УДК 658.26:550.8

Дата подачи статьи: 04.05.21

DOI: $10.15827 / 0236-235 X .135 .484-488$

\title{
Интеллектуальная поддерэка принятия решений в экспертных системах при диагностике заболеваний полости рта
}

\author{
Г.Б. Бурдо 1, д.т.н., зав. кафедрой технологии и автоматизаиии машиностроения, \\ gbtms@yandex.ru \\ Н.А. Семенов ${ }^{1}$, д.т.н., профрессор кафедры информаиионных систем \\ C.H. \\ Ю.В. Аебедева ${ }^{2}$, к.м.н., доиент, ulialebedeva89@gmail.com \\ ${ }_{1}^{1}$ Тверской государственный технический университет, г. Тверь, 170026, Россия \\ 2 Тверской государственный медииинский университет, кафедра хирургической \\ стоматологии и челюстно-лииевой хирургии, г. Тверь, 170100, Россия
}

В настоящее время усилия мировой медицины направлены на раннюю диагностику различных заболеваний. Однако оказание качественной консультативной медицинской помощи больным по месту жительства (сельская местность, районные города) не всегда возможно.

Одним из подходов к расширению квалифицированной, например, стоматологической помощи, по мнению авторов, может быть разработка экспертной системы (в том числе с удаленным доступом), синтезирующей опыт диагностики конкретной группы заболеваний. Экспертные системы находят все большее применение в различных отраслях медицины. Их реализация представляется весьма перспективной и с точки зрения интенсивного развития телемедицины.

Если при ознакомлении с результатами обследований не возникает серьезных методических и технических проблем, то при определении тактики ведения пациента, вырабатываемой в ходе диалога несколькими врачами-экспертами, такие проблемы появляются. В настоящей работе предлагается методика, позволяющая получать согласованное решение врачей по тактике лечения пациента. Методика реализована на основе аппарата нечетких множеств, отличается простотой и не требует специальных знаний в области интеллектуальных систем.

Реализация данной методики позволит врачам-практикам получать обоснованные взвешенные решения, обобщающие коллективные знания, что повышает их точность. Естественно, такие решения можно рассматривать как некую подсказку, а не как безальтернативный вариант. По мере функционирования модели будут уточняться, что повысит эффективность работы экспертной системы.

Ключевые слова: экспертные системы, искусственный интеллект, заболевания полости рта, принятие решений.

Ранняя диагностика различных заболеваний - общемировая проблема. Ситуация осложняется тем, что оказать качественную консультативную медицинскую помощь больным по месту жительства не всегда возможно из-за отсутствия врачей необходимой квалификации [1-4]. Поэтому достаточно актуальна проблема дистанционного взаимодействия врачей с целью выработки тактики ведения пациента. Одним из возможных подходов является использование экспертных систем [5, 6]. Экспертные системы находят все большее применение в различных отраслях медицины, в том числе и при лечении заболеваний полости рта. Их реализация оказывается весьма перспективной и с точки зрения интенсивного развития телемедицины. При ознакомлении с результатами обследований серьезные методиче- ские и технические проблемы могут и не возникнуть, однако при определении тактики ведения пациента, вырабатываемой в ходе диалога несколькими врачами-экспертами (ВЭ), их появление зачастую неизбежно. Поэтому целью исследования является создание интеллектуальных механизмов поддержки принятия решений при лечении пациентов с патологией полости рта, позволяющих формировать рекомендации - подсказки для ВЭ, принимающего окончательное согласованное решение относительно тактики ведения пациента.

\section{Процедуры оценки возможных альтернатив}

Процедура получения согласованного решения может выполняться следующим обра- 
зом. Подбирается группа ВЭ по определенному профилю медицинской направленности. Эксперты могут быть как представителями данного медицинского учреждения, так и внешними. Как правило, группа функционирует в этом составе в течение какого-то определенного периода времени, а не однократно.

Инициатором работы группы является тот ее член, пациента которого обсуждают. Он же и будет лищом, принимающим решение (ЛПР).

Как правило, состав группы может быть следующим: ЛПР, ВЭ, работающие в данной организации, и ВЭ, не работающие в данной организации.

Рекомендуемое число экспертов - 4-6 человек, увеличение состава не дает принципиального повышения точности оценки.

Методика работы коллектива ВЭ под руководством ЛПР состоит в следующем.

1. Присвоение веса (их сумма равна 1 ) с учетом степени ответственности за принимаемое решение и квалификации в данной области медицины (определяются заранее по общепринятым методикам).

2. Установление допустимой нижней границы общей уверенности ВЭ в эффективности определенной тактики ведения пациента (рекомендуется $\geq 0,7)$.

3. Ознакомление ВЭ с результатами обследования пациента (выполняется заранее). Определяется периодичность уточнения тактики лечения.

4. Предложение ВЭ ЛПР возможных тактик ведения пациента (предлагаются заранее).

5. Предложение другими ВЭ возможных тактик ведения пациента.

6. Окончательное определение перечня обсуждаемых альтернативных тактик ведения пациента.

7. Переход к первой альтернативе, ее обсуждение.

8. Оценка альтернативного варианта тактики ведения пациента. Оценивается степень уверенности ВЭ в достижении нужного результата реализацией обсуждаемой тактики в числовой форме (от 0 до 1).

В некоторых случаях удобнее для ВЭ давать оценку не в числовой, количественной форме, а в виде качественной, преобразуя ее в количественную, например, как это показано в таблице.

9. Получение взвешенной оценки общей уверенности ВЭ в достижении результата реализацией рассматриваемой альтернативой с помощью программных средств.

\section{Преобразование оценок степени уверенности}

Converting confidence level assessments

\begin{tabular}{|l|l|l|}
\hline $\begin{array}{l}\text { Качественная } \\
\text { оценка }\end{array}$ & $\begin{array}{l}\text { Интервалы } \\
\text { (справочные) }\end{array}$ & $\begin{array}{l}\text { Принимаемое } \\
\text { значение }\end{array}$ \\
\hline $\begin{array}{l}\text { 1. Абсолютно } \\
\text { не уверен }\end{array}$ & 0 & 0 \\
\hline $\begin{array}{l}\text { 2. Весьма } \\
\text { малая }\end{array}$ & $\begin{array}{l}\text { Свыше } 0 \text { до } \\
0,2\end{array}$ & 0,1 \\
\hline 3. Малая & $\begin{array}{l}\text { Свыше } 0,2 \text { до } \\
0,4\end{array}$ & 0,3 \\
\hline 4. Средняя & $\begin{array}{l}\text { Свыше } 0,4 \text { до } \\
0,6\end{array}$ & 0,5 \\
\hline 5. Высокая & $\begin{array}{l}\text { Свыше } 0,6 \text { до } \\
0,8\end{array}$ & 0,7 \\
\hline $\begin{array}{l}\text { 6. Весьма } \\
\text { высокая }\end{array}$ & $\begin{array}{l}\text { Свыше } 0,8 \text { до } \\
1,0\end{array}$ & 0,9 \\
\hline $\begin{array}{l}\text { 1. Абсолютно } \\
\text { уверен }\end{array}$ & 1,0 & 1,0 \\
\hline
\end{tabular}

10. Переход к следующей альтернативе, повторение пунктов 8 и 9 .

11. Определение даты корректировки хода лечения.

12. Принятие ВЭ - ЛПР тактики ведения пациента, то есть выбор одной из предложенных альтернатив.

\section{База знаний для определения взвешенной оценки}

Следует отдельно пояснить, как определяется степень общей уверенности всех ВЭ. Для этой цели разработана продукционная база знаний с использованием экспертных оценок на основе нечетких множеств. Правила имеют следующую форму:

(ЕСЛИ степень уверенности ВЭ 1 с весом $P_{1}$ равна $\left.A_{1}\right) И$

(ЕСЛИ степень уверенности ВЭ 2 с весом $P_{2}$ равна $\left.A_{2}\right) И$

(ЕСЛИ степень уверенности ВЭ 3 с весом $P_{3}$ равна $\left.A_{3}\right)$ И....

... (ЕСЛИ степень уверенности ВЭ п с весом $P_{n}$ равна $\left.A_{n}\right)$ TO

(взвешенная уверенность экспертов в результате равна $\left.B_{i}\right)$.

Лингвистическая переменная $A_{i}$ имеет три терма: достаточная, средняя, недостаточная (рис. 1).

Лингвистическая переменная $B_{i}$ имеет пять термов: весьма надежная, надежная, сомнительная, ненадежная, весьма ненадежная (рис. 2).

Покажем, как формируется продукционная база нечетких правил определения взвешенной 


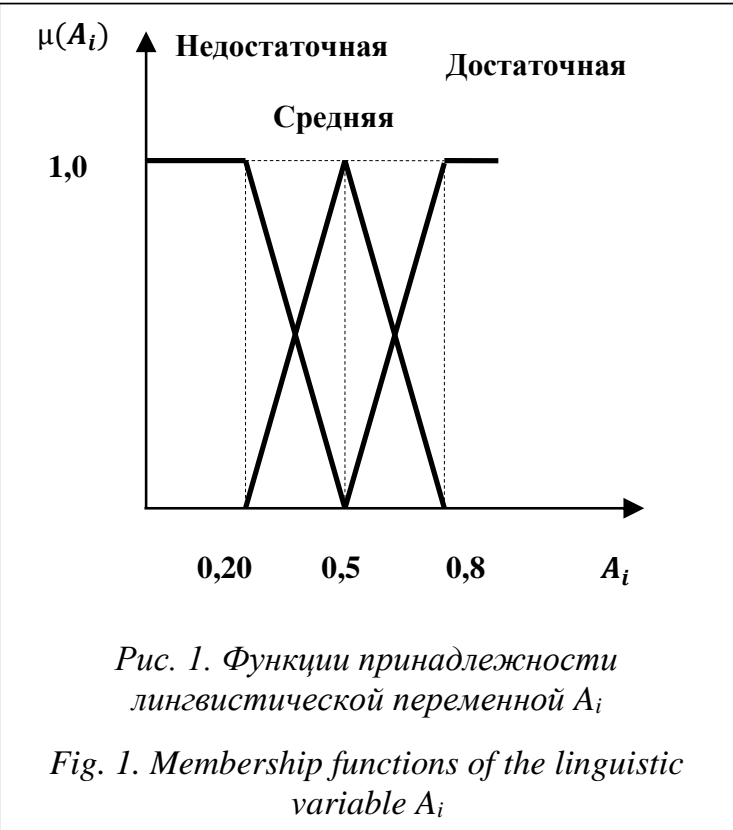

уверенности ВЭ в целесообразности выбранной тактики лечения.

Предложены следующие правила создания моделей.

1. Веса ВЭ $P_{i}$ принимаются из ряда чисел, кратных $k_{i}$ раз $\left(k_{i}-\right.$ целое число, равное или большее 1) минимальному из них:

$P_{\text {min }}=\min \left\{P_{1}, P_{2}, \ldots, P_{i}, \ldots, P_{n}\right\}$.

2. Базовым является подусловие для ВЭ с минимальным значением веса $P_{\min }$.

3. Осуществляется преобразование подусловий по введенному правилу, когда одно подусловие для ВЭ с весом, большим $P_{\min }$, заменяется $k_{i}$ подусловиями, соединенными оператором «И».

Например, исходное подусловие имеет вид: (ЕСЛИ уверенность ВЭ і с весом $\left(k_{i} \times P_{\min }\right)$ равна $\left.A_{i}\right)$ И...

Заменяющие его $k_{i}$ подусловия:

(ЕСЛИ уверенность ВЭ і с весом $P_{\min }$ равна $A_{i}$

ЕСЛИ уверенность ВЭ і с весом $P_{\min }$ равна $A_{i} h$

... ЕСЛИ уверенность ВЭ $i$ с весом $P_{\min }$ равна $\left.A_{i}\right)$ И...

То есть исходное подусловие разбивается на $k_{i}$ подусловий.

4. С учетом п. 2 записываются нечеткие продукционные правила определения взвешенной уверенности экспертов.

5. В продукционном правиле взаимоисключаются подусловия, содержащие значения лингвистической переменной $A_{i}$ (достаточная $\Leftrightarrow$ недостаточная).

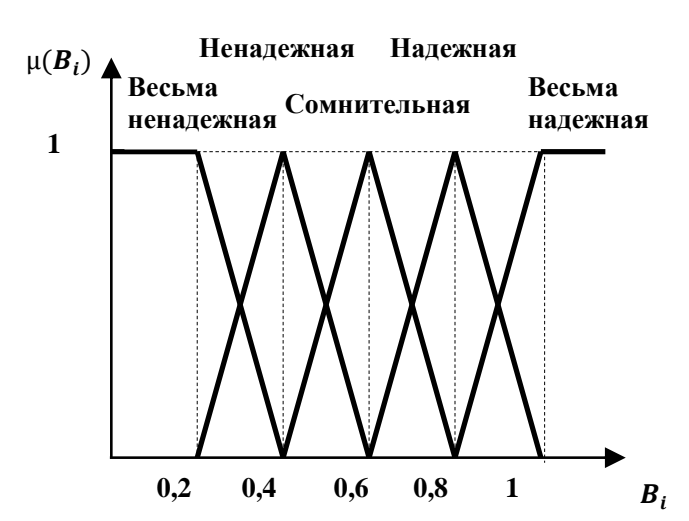

Рис. 2. Функции принадлежности лингвистической переменной $B_{i}$

Fig. 2. Membership functions of the linguistic variable $B_{i}$

Например, исходное правило:

(ЕСЛИ уверенность ВЭ 1 - достаточная И

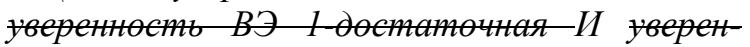
ноен ВЭ 2 педоенан И уверенность ВЭ 3 - достаточная И уверенность ВЭ 3 - достаточная И уверенность ВЭ 4 - средняя) TO...

Преобразованное правило:

(ЕСЛИ уверенность ВЭ 1 - достаточная И уверенность ВЭ 3 - достаточная И уверенность ВЭ 3 - достаточная И уверенность ВЭ 4 - средняя) ТО...

Очевидно, что в результате имеем продукционные правила, содержащие только смежные значения лингвистической переменной $A_{i}$.

Были разработаны продукционные правила для четырех ВЭ в группе.

C помощью экспертных оценок сформированы следующие нечеткие продукционные правила, определяющие значение лингвистической переменной $B_{i}$ (взвешенная уверенность ВЭ).

1. ЕСЛИ все подусловия содержат значение лингвистической переменной $A_{i}$ - достаточная ТО значение лингвистической переменной $B_{i}-$ весьма надежная.

2. ЕСЛИ все подусловия содержат значение лингвистической переменной $A_{i}$ - недостаточная ТО значение лингвистической переменной $B_{i}-$ весьма ненадежная.

3. ЕСЛИ число подусловий со значением лингвистической переменной $A_{i}$ - средняя (превышает число подусловий со значением лингвистической переменной $A_{i}$ - достаточная или недостаточная) ИЛИ (не более, чем на одно меньше числа подусловий со значением лингви- 
стической переменной $A_{i}$ - достаточная или недостаточная) ТО лингвистическая переменная $B_{i}-$ сомнительная.

4. ЕСЛИ (число подусловий со значением лингвистической переменной $A_{i}-$ достаточная или недостаточная более, чем на одно превьшает число подусловий со значением лингвистической переменной $A_{i}$ - средняя) ТО лингвистическая переменная $B_{i}-$ надежная или ненадежная соответственно.

Например: (ЕСЛИ уверенность ВЭ 1 - достаточная И уверенность ВЭ 3 - достаточная И уверенность ВЭ 3 - достаточная И уверенность ВЭ 4 - средняя) ТО значение лингвистической переменной $B_{i}$ - надежная.

Фазилогическая конъюнкция подусловий выполняется по правилу минимума, нахождение функций совместной принадлежности - по правилу нечеткой импликации Мамдани. Дефазификация взвешенной оценки уверенности экспертов (результат) выполняется по центроидному методу $[7,8]$.

\section{Заключение}

Методика была реализована в среде MATLAB [8]. Ее использование позволит врачам-практикам получать обоснованные взвешенные решения, обобщающие коллективные знания врачей, что повышает их точность. Естественно, такие решения можно рассматривать как некую подсказку врачу, а не как безальтернативный вариант. По мере функционирования системы модели будут уточняться, что повысит эффективность работы экспертной системы.

\section{Лumepamypa}

1. Давыдов А.Б. Диагностика и лечение карцином полости рта. Тверь, 2007. 272 с.

2. Чиссов В.И., Старинский В.В., Петрова Г.В. Злокачественные новообразования в России в 2011 году (заболеваемость и смертность). М., 2013. 289 с.

3. Лебедев С.Н., Волков С.И., Лебедева Ю.В., Красовский П.В. Онкостоматология. Анализ показателей местного статуса пациентов с заболеваниями слизистой оболочки полости рта, ассоциированными с гипер(дис) кератозом и/или дисплазией эпителия // Современная стоматология: проблемы, задачи, решения: сб. тр. 2019. С. 82-87.

4. Поддубная И.В. Онкология: справочник практического врача. М.: МЕДпресс-информ, 2009. $768 \mathrm{c}$.

5. Рыбина Г.В. Основы построения интеллектуальных систем. М.: Финансы и статистика; Инфра-М, 2010. 432 с.

6. Гаврилова Т.А., Хорошевский В.Ф. Базы знаний интеллектуальных систем. СПб: Питер-пресс, 2016. $162 \mathrm{c}$.

7. Заде Л. Понятие лингвистической переменной и ее применение к понятию приближенных решений. М.: Мир, 1976. 165 с.

8. Дьяков В., Круглов В. Математические пакеты расширения MATLAB. СПб: Питер, 2001. 480 с.

Software \& Systems

DOI: $10.15827 / 0236-235 X .135 .484-488$

\section{Intelligent decision support in expert systems in the diagnosis of oral cavity diseases}

G.B. Burdo ${ }^{1}$, Dr.Sc. (Engineering), Head of Department, gbtms@yandex.ru

N.A. Semenov ${ }^{1}$, Dr.Sc. (Engineering), Professor

S.N. Lebedev ${ }^{2}$, Ph.D. (Medics), Associate Professor, lebedev_s@tvergma.ru

Yu.V.Lebedeva ${ }^{2}$, Ph.D. (Medics), Associate Professor, ulialebedeva89@gmail.com

${ }^{1}$ Tver State Technical University, Tver, 170026, Russian Federation

${ }^{2}$ Tver State Medical University, Tver, 170100, Russian Federation

Abstract. Nowadays, the efforts of the world medicine focus on the early diagnosis of various oncological diseases. The situation is complicated by the fact that the provision of high-quality advisory medical care to such patients at the place of residence (rural areas, district cities) is not always possible due to the lack of doctors with the necessary qualifications. For example, even in the central part of Russia, specialized oncological institutions are located only in regional centers. 
According to the authors, one of the approaches to expanding qualified dental care may consist in the development of an expert system (including remote access) that synthesizes the experience of diagnosing this group of diseases. Expert systems are more commonly used in various medicine branches. Their implementation is also very promising in terms of intensive development of telemedicine. If there are no serious methodological and technical problems when studying the results of examinations, then certain problems appear when determining the tactics of patient management developed during the dialogue of several expert doctors.

This paper proposes a method for obtaining a coordinated decision of doctors on the tactics of treating a patient. The method is implemented based on the fuzzy sets device. It is simple and does not require special knowledge from doctors in the field of intelligent systems.

The implementation of this method will allow practitioners to obtain informed and balanced decisions that summarize the collective knowledge of doctors that increases their accuracy. Naturally, such decisions can be considered as a kind of hint to a doctor and not as an alternative option. As the system functions, the models will be refined, which will increase the expert system efficiency.

Keywords: expert systems, artificial intelligence, oral diseases, decision-making.

\section{References}

1. Davydov A.B. Diagnosis and Treatment of Oral Carcinomas. Tver, 2007, 272 p. (in Russ.).

2. Chissov V.I., Starinsky V.V., Petrova G.V. Malignant Neoplasms in Russia in 2011 (Morbidity and Mortality). Moscow, 2013, 289 p. (in Russ.).

3. Lebedev S.N., Volkov S.I., Lebedeva Yu.V., Krasovsky P.V. Oncostomatology. Analysis of local status indicators of patients with diseases of the oral mucosa associated with hyper(dys) keratosis and/or epithelium dysplasia. Proc. Modern Dentistry: Problems, Tasks, Solutions, 2019, pp. 82-87 (in Russ.).

4. Poddubnaya I.V. Oncology: a Practicing Physician's Guide. Moscow, 2009, 768 p. (in Russ.).

5. Rybina G.V. The Basics of Building Intelligent Systems. Moscow, 2010, 432 p. (in Russ.).

6. Gavrilova T.A., Horoshevsky V.F. Knowledge Bases of Intelligent Systems. St. Petersburg, 2016, 162 p. (in Russ.).

7. Zade L. The Concept of a Linguistic Variable and Its Application to the Concept of Approximate Solutions. Moscow, 1976, 165 p. (in Russ.).

8. Dyakov V., Kruglov V. MATLAB Math Extension Packages: A Special Reference. St. Petersburg, 2001, 480 p. (in Russ.).

\section{Для цитирования}

Бурдо Г.Б., Семенов Н.А., Аебедев С.Н., Аебедева Ю.В. Интемлектуальная поддержка принятия решений в экспертных системах при диагностике заболеваний полости рта // Программные продукты и системы. 2021. Т. 34. № 3. C. 484-488. DOI: 10.15827/0236-235X.135.484-488.

\section{For citation}

Burdo G.B., Semenov N.A., Lebedev S.N., Lebedeva Yu.V. Intelligent decision support in expert systems in the diagnosis of oral cavity diseases. Software \& Systems, 2021, vol. 34, no. 3, pp. 484-488 (in Russ.). DOI: 10.15827/0236-235X.135.484-488. 